\title{
Correlation between ribonucleoside-diphosphate reductase and three replication proteins in Escherichia coli
}

\author{
M Antonia Sánchez-Romero, Felipe Molina, Alfonso Jiménez-Sánchez ${ }^{*}$
}

\begin{abstract}
Background: There has long been evidence supporting the idea that RNR and the dNTP-synthesizing complex must be closely linked to the replication complex or replisome. We contributed to this body of evidence in proposing the hypothesis of the replication hyperstructure. A recently published work called this postulate into question, reporting that $\mathrm{NrdB}$ is evenly distributed throughout the cytoplasm. Consequently we were interested in the localization of RNR protein and its relationship with other replication proteins.

Results: We tagged NrdB protein with $3 \times F$ FAG epitope and detected its subcellular location by immunofluorescence microscopy. We found that this protein is located in nucleoid-associated clusters, that the number of foci correlates with the number of replication forks at any cell age, and that after the replication process ends the number of cells containing NrdB foci decreases.

We show that the number of $\mathrm{NrdB}$ foci is very similar to the number of SeqA, DnaB, and DnaX foci, both in the whole culture and in different cell cycle periods. In addition, interfoci distances between NrdB and three replication proteins are similar to the distances between two replication protein foci.

Conclusions: $\mathrm{NrdB}$ is present in nucleoid-associated clusters during the replication period. These clusters disappear after replication ends. The number of these clusters is closely related to the number of replication forks and the number of three replication protein clusters in any cell cycle period. Therefore we conclude that NrdB protein, and most likely RNR protein, is closely linked to the replication proteins or replisome at the replication fork. These results clearly support the replication hyperstructure model.
\end{abstract}

\section{Background}

Initiation of chromosome replication is a key and tightly regulated event in the $E$. coli cell cycle. Initiation starts by the binding of DnaA protein to the oriC sequence. This facilitates DNA strand opening, and allows the subsequent loading of the primosomal proteins DnaB helicase and DnaG primase, and, finally, the DNA polymerase III holoenzyme, forming the replisomes that will lead to bidirectional chromosome replication [1]. DNA synthesis requires a balanced supply of the four dNTPs in all living cells. NDP reductase (RNR) is an essential enzyme for the synthesis of these precursors in most organisms [2,3]. RNR is a tetramer made of two homodimers - subunit R1 coded by the $n r d A$ gene and

\footnotetext{
*Correspondence: a.jimenezsanchez@gmail.com

Departamento de Bioquímica y Biología Molecular y Genética, Universidad de Extremadura, E06080, Badajoz, Spain
}

subunit R2 coded by the $\operatorname{nrdB}$ gene [2]. A body of evidence supports the idea that RNR and the dNTPsynthesizing complex must be closely linked to the replication complex or replisome [4]. The dNTP concentrations required for optimal in vivo DNA synthesis appear to be four times higher than measured cellular pools $[4,5]$. Furthermore, allosteric regulation of RNR activity, controlled by the ATP to dATP ratio, will not function in free cytoplasmic enzymes where the ATP pool is 20 times greater than dATP [6], so that this regulation can only be achieved in a highly concentrated dATP environment not present in whole cells [7]. Close proximity of the dNTP-synthesizing complex to the replisome inside an enclosed structure would provide the required precursors for DNA synthesis in the place where they are needed and at the required concentrations. Other observations supporting the suggested 
compartmentation of precursors show that permeabilized E. coli cells incorporate dNMPs into DNA more efficiently than dNTPs, and inhibition of nucleoside diphosphate kinase inhibits incorporation of both dNMPs and dNTPs [8]. Furthermore, a direct interaction of RNR with protein $\mathrm{MreB}$, involved in chromosome segregation, and with protein DnaN, the $\beta$-clamp subunit of DNA polymerase III, have been described by Butland et al. [9]. Interactions between dNTP synthesis and proteins involved in DNA replication have also been shown in T4 [8,10-12] and in eukaryotic cells $[13,14]$.

In a previous paper [15] we showed that the thermosensitive RNR protein coded by allele $n r d A 101$, which loses its activity in vitro after a few seconds [16], in vivo maintains its activity for more than $160 \mathrm{~min}$. Bacterial strains with this mutant allele perform replication fork reversal (RFR), as thermosensitive replication proteins do at the restrictive temperature, unrelated to the inhibition of deoxynucleotide synthesis [17]. Furthermore, the growth of these mutant cells at the non-permissive temperature also affects nucleoid organization and chromosome segregation in a manner independent of the protein's enzymatic activity [18], which might be explained by the physical interaction of RNR with protein MreB [9]. These results point towards the requirement of a precise structure of RNR, in addition to its reductase activity, to maintain an active replication fork and to allow chromosome segregation and a precise cell division [18]. Together, this evidence suggests the presence of a replication hyperstructure where all the functions required for precursor biosynthesis and chromosome replication and segregation would be assembled in a higher order structure $[15,19,20]$.

This hyperstructure should require RNR, and consequently the dNTP-synthesizing complex, to be associated with replication forks. It has been suggested that this replication hyperstructure is disrupted when the supply of nucleotides is limited or when thymidylate synthetase is mutated [21]. Recently, RNR has been suggested to be a central element of this hyperstructure [22]. However, a later study indicated that RNR may be evenly distributed throughout the cell since no discrete foci were observed [23]. Consequently, we were interested in establishing whether RNR is found in discrete foci or is dispersed throughout the cell, and, if present, whether those foci were in close proximity to the replication complex.

Using fluorescence microscopy and epitope tagged proteins, we determined the location of RNR and three replication proteins in slowly growing cultures of $E$. coli. We observed discrete foci formation of the NrdB::3 $\times$ FLAG protein. When this tagged protein was analyzed together with immunolabeled SeqA protein, which binds newly replicated hemimethylated DNA and consequently defines the localization of replication forks [24], with DnaB helicase, or with DnaX $\tau$ subunit, we observed similar average numbers of foci of the four tagged proteins in the full bacterial population, and we also found similar numbers of the four foci when cells were grouped according to the number of predicted replication forks. Furthermore, inter-foci distances between NrdB and each of the three selected replication proteins were similar to or even less than those between replication proteins. These results support a coordinated organization of NrdB protein, and consequently RNR protein, with other replication proteins, and provide support for the replication hyperstructure model.

\section{Results}

\section{$\mathrm{NrdB}$ is found in discrete foci that correlate with the} number of replication forks

A recently published work showed that immunolabeled RNR does not form discrete foci in E. coli suggesting that this protein might be dispersed throughout the cell [23]. This result called into question our interpretation of the results of our previous studies as indicating a close association of RNR with the replisome $[15,17,18]$. Consequently, we were interested in confirming or rejecting this interpretation of our data by analyzing the capability of immunolabeled $3 \times$ FLAG-tagged RNR to form foci.

Analysis of slowly growing $E$. coli cells greatly facilitates interpretation of replication data due to reduced overlap of consecutive cell cycles, and the lower number of replication forks per cell. Faster growth rates cause increasing numbers of overlapping replication cycles, and consequently more replication forks per cell. Furthermore, two or more replication forks can aggregate and their co-localization correlates with the overlapping of replication cycles, and therefore the discrepancy between the number of forks and foci per cell increases with the growth rate in wild-type cells [25-27]. In the present work cells were grown in glycerol minimal medium at $37^{\circ} \mathrm{C}$ and cell cycle parameters were studied by flow cytometry (data not shown) as described in Methods. Insertion of the $3 \times$ FLAG epitope at the $C$ terminus of $\mathrm{NrdB}$ did not change the cell cycle parameters from those of the parental strain (Figure 1), which suggests that the tagged RNR protein has no detrimental effect upon bacterial growth and chromosome replication. Insertion of the HA epitope at the $\mathrm{C}$ terminus of DnaB helicase or $\tau$ subunit DNA polymerase, however, increased the replication period to $110 \mathrm{~min}$ and cut the $D$ period down to $4 \mathrm{~min}$ in both strains. These results make it possible to obtain a complete description of the proportion of cells with their expected chromosome structure and the number of replication 


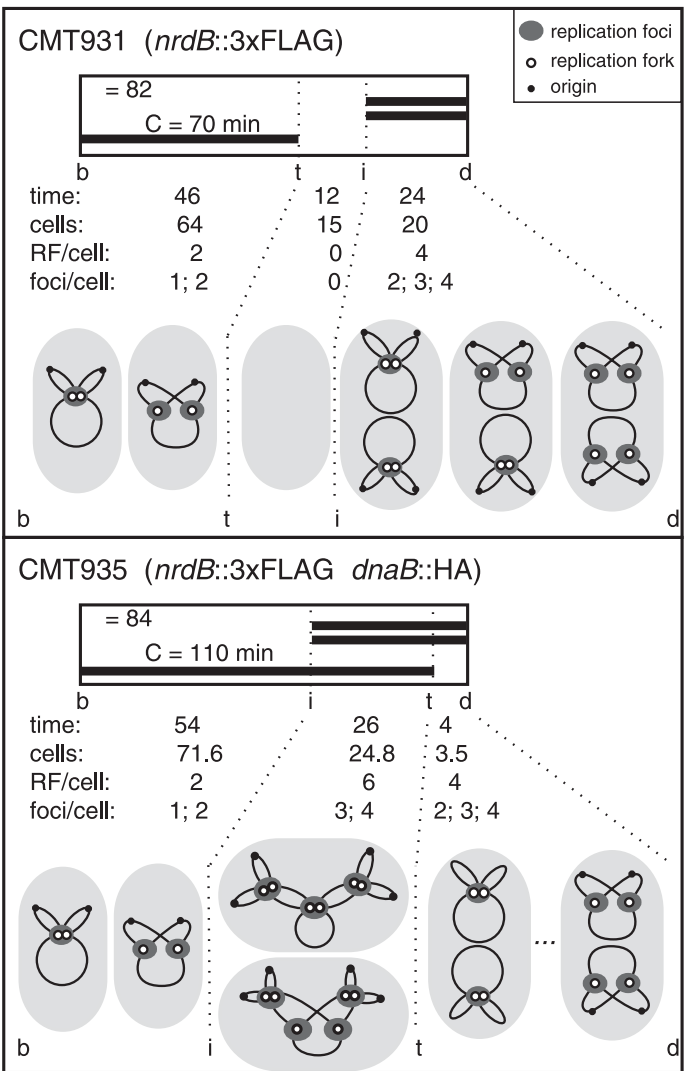

CMT936 (nrdB::3xFLAG dnaX::HA)
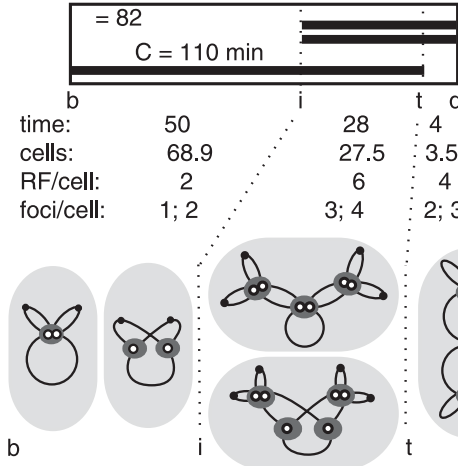

$\therefore \quad 6: 4$

$\therefore \quad 3 ; 4 \quad 2 ; 3 ; 4$
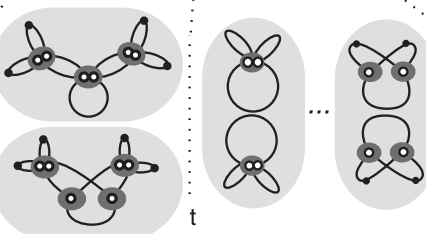

Figure 1 Cell cycle events and chromosome structure. Cell cycle events and chromosome shapes relative to cell age in strains CMT931, CMT935, and CMT936 growing in glycerol minimal medium at $37^{\circ} \mathrm{C}$. $\tau$ is the doubling time in minutes. $\mathrm{C}$ is the time in minutes required to replicate the chromosome. Thick bars represent replicating chromosomes. Time refers to the length in minutes of each of the three periods determined by cell birth $(b)$, initiation of replication ( $i)$, termination of replication $(t)$, and cell division $(d)$. cells is the frequency of cells, in percentages, in each of the three periods. RF/cell is the number of replication forks per cell, and foci/ cell is the expected number of foci per cell of a fluorescent replication protein depending on the association state of the sister replication forks, in each of the three cell cycle periods. The inset shows the symbols for the origin of replication (small black circle), for each replication fork (open circle), and for the foci of an immunostained replication protein (grey oval). forks. The expected number of foci for any replication protein was deduced by considering the association of the two sister replication machineries as described elsewhere [26] (Figure 1).

Fluorescence microscopy of CMT931 immunolabeled with Cy3 conjugated anti-FLAG antibody showed that all cells contained from one to four discrete foci (Figure 2 ), and no diffuse fluorescence could be seen in any cell. Cells were also stained with Hoechst 33258 to detect bacterial nucleoids. The micrographs obtained showed that all NrdB foci were localized within the nucleoid boundary or in its periphery. Consequently we can conclude that RNR is essentially concentrated in discrete clusters and not spread throughout the cell, and that these clusters are associated with the nucleoid.

A higher level of information can be achieved by comparing the number of foci per cell with the number of replication forks in each period of the cell cycle. Given that cell length increases exponentially throughout the cell cycle, and notwithstanding the variation in length at birth and at division [28,29], it can be used to estimate the age of a cell. We therefore used cell length to sort the bacteria into the three periods determined by the initiation and termination of chromosome replication. In each group of cells, the number of $\mathrm{NrdB}$ foci corresponded very closely to what was predicted for the number of replication forks (Figure 3). This high level of equivalence between $\mathrm{NrdB}$ foci and the replication forks suggests a close relationship between the RNR and the replisome.

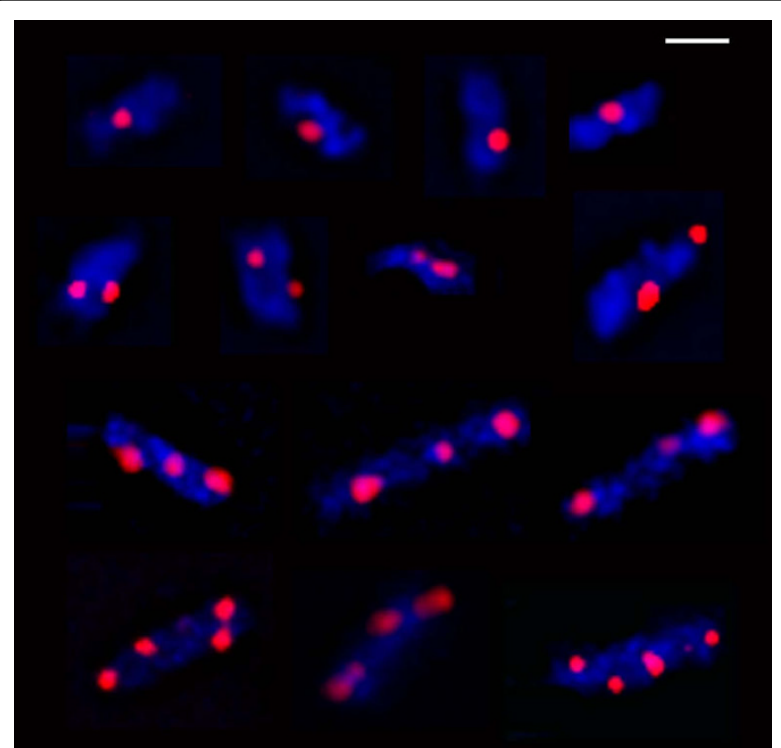

Figure 2 Fluorescence microscopy of tagged-NrdB cells Fluorescence microscopy images of cells of CMT931 (nrdB:3xFLAG) immunolabeled with Cy3 conjugated anti-FLAG antibody (red) and

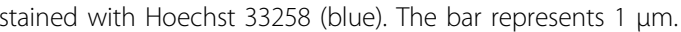




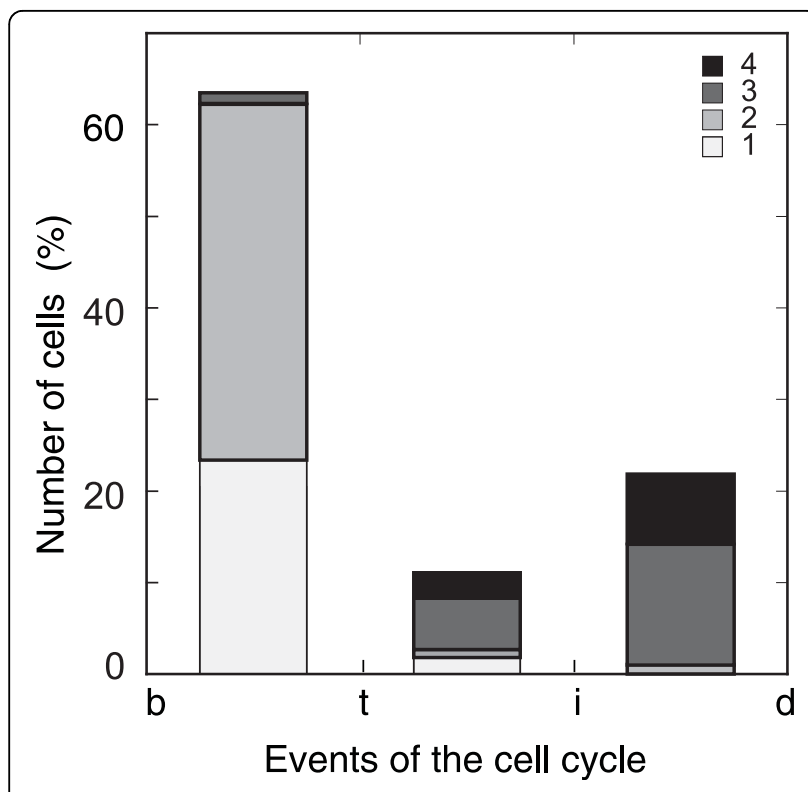

Figure $3 \mathrm{NrdB}$ foci and replication forks. Percentage of cells containing different numbers of NrdB::3XFLAG foci per cell of strain CMT931, distributed into the three periods of the cell cycle defined as from birth $(b)$ to termination of replication $(t)$, from termination to initiation ( $i)$, and from initiation to cell division $(d)$, as described in Figure 1. Number of cells analyzed was 426 . The inset shows the colour code for the four numbers of foci per cell.

A hypothesized connection between RNR and the replication fork would predict that NrdB foci should decrease after replication ends. In order to investigate this issue, we used a synchronized culture for initiation of chromosome replication of strain CMT934 which includes allele $d n a C 2$ together with $n r d B:: 3 \times$ FLAG. Fluorescence microscopy of these synchronized cells showed that, when they were replicating, 80 per cent of cells had one or two NrdB foci (Figure 4). The 20 per cent without foci could denote cells that did not initiate replication during the activation of the replication period. After replication ended, the number of cells without NrdB foci increased threefold, and the number of cells containing NrdB foci decreased. These results show that, once replication forks are expected to have finished [30], the number of $\mathrm{NrdB}$ foci, and consequently RNR assembly, also diminishes, thus supporting the proposed connection between the RNR and the replication fork.

NrdB foci are found in essentially the same numbers as foci from SeqA clusters, DnaB helicase, and DNA polymerase III $\tau$ subunit

We then were interested in comparing the average number of $\mathrm{NrdB}$ foci in an exponentially growing culture with that of three proteins associated with replication forks: SeqA protein, known to bind newly replicated DNA and, therefore, the replication fork [24], and two interacting components of the replisome - DnaB

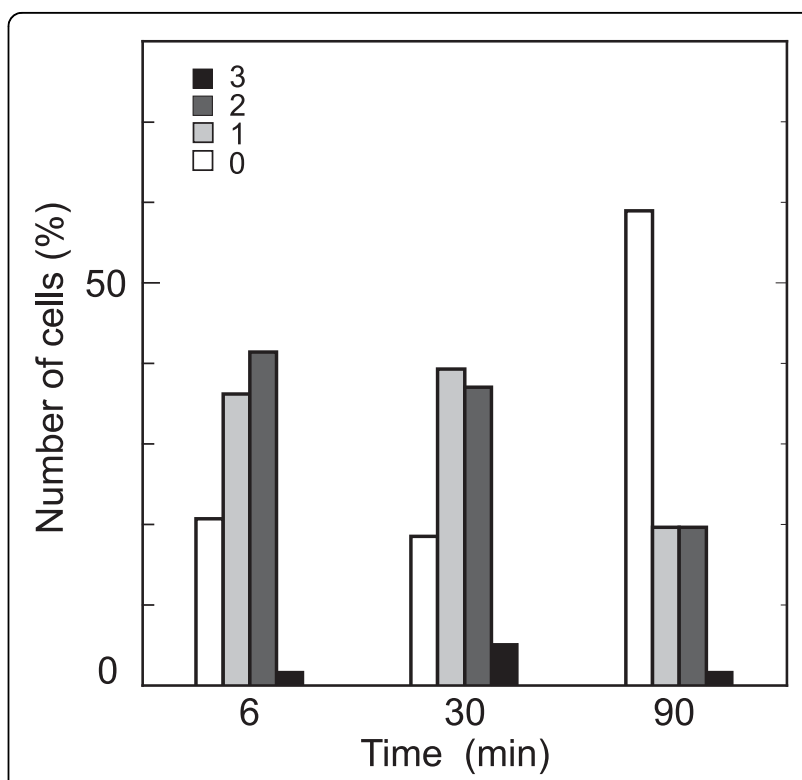

Figure 4 Analysis of $\mathrm{NrdB}$ foci during synchronous replication Number of NrdB foci in a CMT934 (nrdB::XFLAG dnaC2) culture after the three-step temperature shifts for synchronous initiation of one round of chromosome replication. Samples were withdrawn at 6 , 30 , and $90 \mathrm{~min}$ after the beginning of the $6 \mathrm{~min}$ period at $30^{\circ} \mathrm{C}$ to allow one initiation round. Number of cells analyzed was 261 . The inset shows the colour code for the number of foci per cell.

helicase and the DNA polymerase III $\tau$ subunit coded by the $d n a X$ gene. For this purpose, we constructed strain CMT935, containing a C-terminal HA-tagged DnaB helicase and the $\mathrm{NrdB}:: 3 \times$ FLAG protein, and strain CMT936, containing a C-terminal HA-tagged DnaX subunit and the $\mathrm{NrdB}:: 3 \times \mathrm{FLAG}$ protein.

Immunolabeling of RNR together with any of the aforementioned proteins revealed the presence of foci for every protein (Figure 5). Counts of the numbers of each kind of foci per cell gave a clear-cut pattern of similarity between $\mathrm{NrdB}$ foci and those of the replication proteins (Figure 6). All four proteins were represented in the culture by $1,2,3$, and 4 foci, with 1 and 2 foci being predominant in consonance with the number of predicted foci (Figure 1). Furthermore, the number of foci per cell of every replication protein was very similar to the number of NrdB foci. From these results it seems to be evident that the RNR protein is closely related to the replication proteins, and suggests that it should be mainly concentrated at the replication forks.

It is worth noting that those cells containing 2, 3, or 4 $\mathrm{NrdB}$ foci, which have a high consistency with the number of DnaB and DnaX foci, were found to have the same number or more of those foci than of SeqA foci (Figure 6A). The number of DnaB helicase and DnaX $\tau$ subunit foci, also greater than that of SeqA foci, could be explained by pre-replication assembly of the 


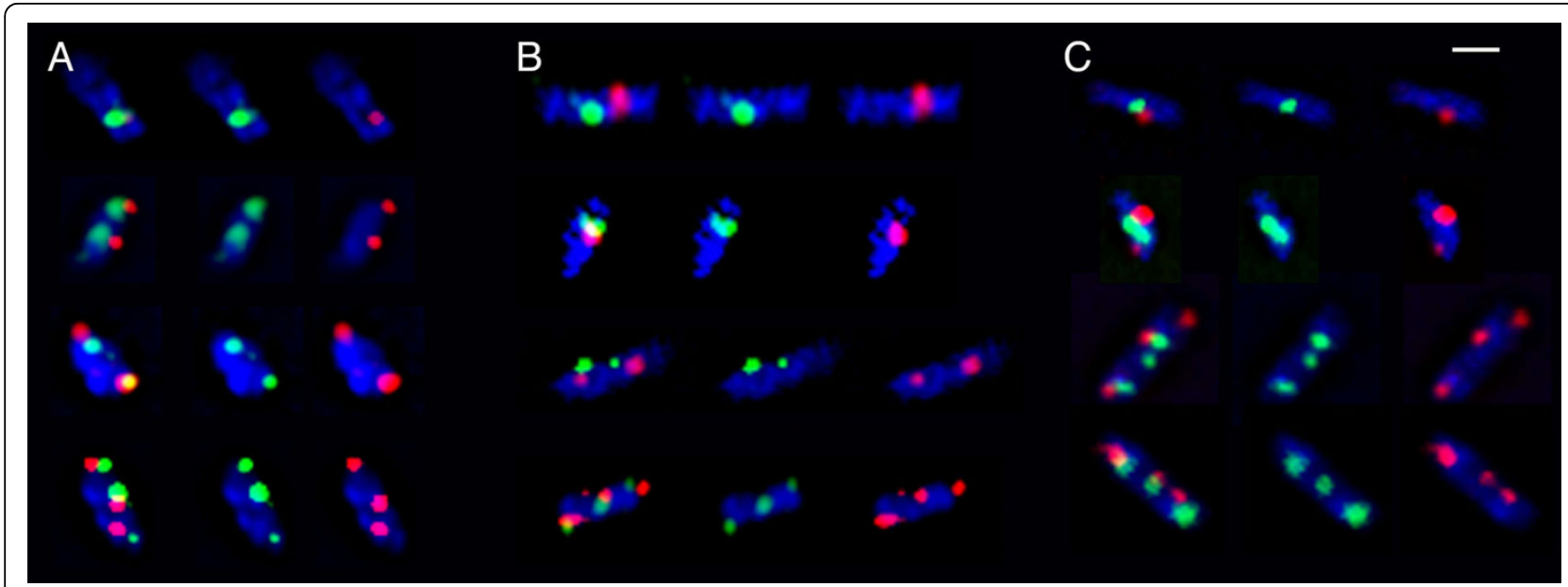

Figure 5 Fluorescence microscopy of tagged-NrdB and SeqA, or DnaB, or DnaX cells. Fluorescence microscopy images of cells of CMT935 (nrdB:3xFLAG dnaB::HA) (A) or CMT936 (nrdB:3XFLAG dnaX::HA) (B) immunolabeled with Cy3-anti-FLAG (red) and FITC-anti-HA (green), or CMT931 (nrdB::3XFLAG) immunolabeled with Cy3 conjugated anti-FLAG (red) and FITC-anti-SeqA (green) antibodies (C). Cells were also stained with Hoechst 33258 for nucleoid visualization. Each group of cells shows nucleoid (blue) and, from left to right: both green and red, only green, and only red fluorescence. The bar represents $1 \mu \mathrm{m}$.

replisome proteins [31]. Likewise, the greater number of $\mathrm{NrdB}$ foci relative to those of SeqA can be explained by assuming that this protein was also pre-assembled together with other replisome proteins before replication initiated.

\section{The number of NrdB foci throughout the cell cycle} matches with those of SeqA, DnaB helicase, and $\tau$ subunit To analyze the foci distribution during the cell cycle, we arranged the cells into the three cycle periods determined by the initiation and termination of chromosome replication. For each group of cells, the numbers of foci of each of the four tagged proteins were determined. The data displayed in Figure 7 reveal very strong agreement between the number of NrdB foci (Figure 7, lower panels) and the numbers of foci of each replication protein (Figure 7, upper panels) in each cell cycle period. These results provide further evidence for the strong correlation between the RNR and the replication proteins.

There was only a slight inconsistency observed in the relationship between $\mathrm{NrdB}$ and SeqA foci with there being more of the former than of the latter. As mentioned in the previous section, this inconsistency could be ascribed to pre-replication assembly of the replication proteins.

NrdB foci localize near SeqA, DnaB helicase, and $\tau$ subunit foci

Notwithstanding the coincidence between the number of $\mathrm{NrdB}$ foci and replication protein foci being a good indication of the association between RNR and the replisome proteins, a further property of this relationship should be the close subcellular location of the associated proteins. The possible nearness of RNR to the replication proteins was determined by measuring the distance between the pairs of foci. In particular, we measured inter-foci distances between $\mathrm{NrdB}$ foci and SeqA, or DnaB helicase, or DnaX $\tau$ subunit foci in cells containing the same number of both tagged proteins to obviate pre-assembly generated foci. For a correct understanding of the observed dimensions, and as a control of closeness between proteins known to be at the replication fork, we also measured distances between SeqA and DnaB helicase foci and between SeqA and $\tau$ subunit foci. The observed inter-foci distances between $\mathrm{NrdB}$ and each of the replication proteins gave very similar values to those found between SeqA and DnaB or DnaX foci (Table 1). Interfoci distances were measured from the centres of foci. As foci have an average diameter of $0.26 \mu \mathrm{m}$, one can deduce from the data in Table 1 an overlap of foci in 90, 52, and 80 per cent of foci pairs of NrdB with SeqA, DnaB, and DnaX, respectively. From the same table one can deduce that DnaB and DnaX protein foci, known to be at the replication fork, overlap with SeqA foci in 73 and 78 per cent of the studied pairs of foci, respectively.

To rule out the a priori not unlikely possibility of random localization, we studied inter-foci distances between $\mathrm{NrdB}$ and replication protein foci relative to the number of pairs of foci per nucleoid length. The rationale of this approach was that, with a random distribution of foci, the higher the number of foci per cell length the smaller the inter-foci distances. Inter-foci distances between NrdB and DnaX $\tau$ subunit in cells with different numbers of foci per nucleoid length are shown 


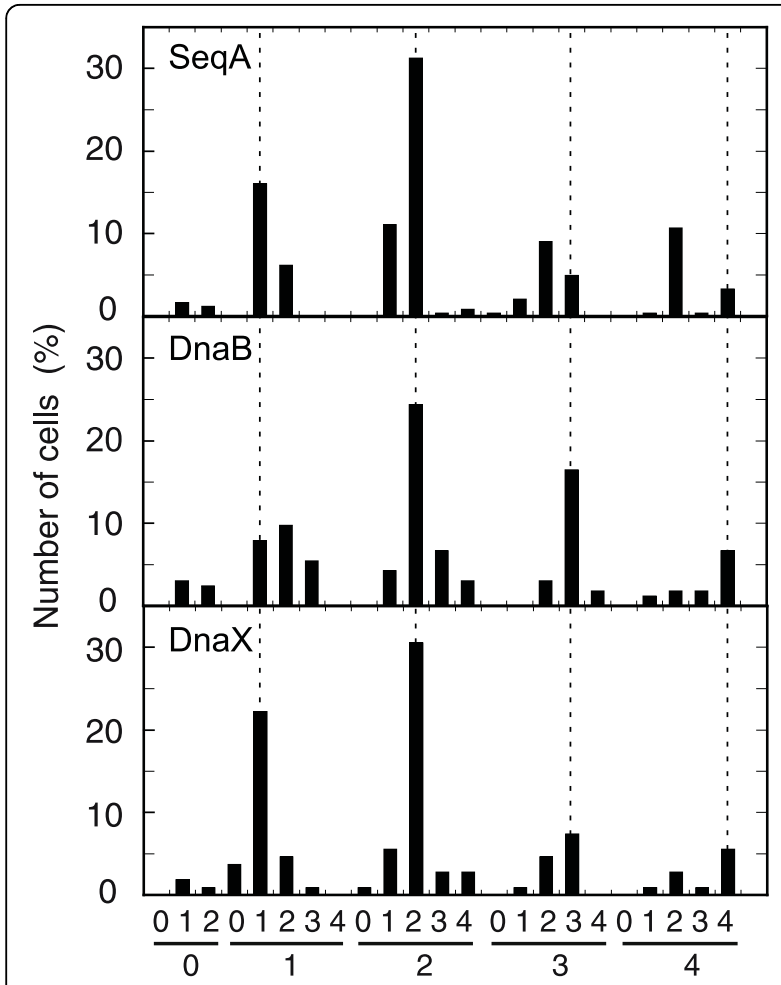

Number of foci (SeqA, or DnaB, or DnaX / NrdB)

Figure $6 \mathrm{NrdB}$ foci related to SeqA, DnaB, and DnaX foci Frequency of the number of foci of NrdB together with SeqA fluorescent proteins in strain CMT931 (nrdB:3xFLAG), or with DnaB helicase in strain CMT935 (nrdB::3×FLAG dnaB::HA), or with DNA polymerase III $\tau$ subunit in strain CMT936 ( $n$ rdB:3 $\times$ FLAG dnaX::HA) in mid log cells growing in glycerol minimal medium at $37^{\circ} \mathrm{C}$.

Numbers in the upper position of the horizontal axis are the number of foci of any of the three replication proteins present in a cell containing the number of $\mathrm{NrdB}$ foci indicated by the number in the lower position. Dotted lines identify cells with the same number of both foci. Numbers of cells analyzed were 129, 172, and 110, respectively.

in Figure 8. The data show that distances between $\mathrm{NrdB}$ and $\tau$ foci are independent of foci compaction, and hence can not be explained by a random distribution. Very similar results were obtained when this analysis was performed with $\mathrm{NrdB}$ and $\mathrm{DnaB}$ foci, or with $\mathrm{NrdB}$ and SeqA foci (data not shown). Consequently, these results establish the high degree of nearness of the proteins studied, and confirm that RNR protein can not be farther away from the replisome than are other replication proteins.

\section{Discussion}

According to Mathews' and our own work, we had proposed that a replication hyperstructure should be the association of all the proteins required for replication, i. e., the replisome together with the dNTP-synthesizing complex and most likely with proteins required for chromosome segregation, all included in some membrane structure $[4,15,18-20]$. This model would require RNR protein to be localized very close to the replication proteins. This should cause the fluorescence of immunolabeled RNR protein to be concentrated in discrete foci, and these foci should also be present in similar numbers to other fluorescent replication protein foci and in close proximity to them.

A recent work had put the likelihood of this model into doubt by showing that immunolabeled RNR did not appear in foci but seemed to be dispersed throughout the cell [23]. We were therefore interested in a further study to confirm or invalidate this result and in consequence our model.

We first decided to label RNR protein by tagging a short epitope to the $\mathrm{C}$-terminus of $\mathrm{R} 2$ subunit as an alternative to the more severe binding of a fluorescent protein, which would disturb a likely higher structure. The correct functionality of this tagged protein was verified by checking that growth rate and replication parameters were the same as the parental strain. When the strain CMT931, containing the NrdB::3×FLAG tagged polypeptide, was immunolabeled with a specific antiFLAG antibody and observed by fluorescence microscopy, all cells were perceived to bear from one to four discrete foci. The difference with the findings in the work of Watt's group might be ascribable to the epitope used to tag the protein [23]. In that work they used a derivative of GFP to tag NrdB. The RFP or GFP $(\approx 34$ $\mathrm{kDa}$ ) fusion can compromise the full function of the target protein that can change the pattern of its localization [32]. This possible alteration is more crucial when a higher order structure is being proposed for RNR.

Cell size was also measured, and cells were distributed by their length into the three periods of a cell cycle, determined by the initiation and termination of replication, in order to study the relationship between predicted fork numbers in each period and the observed foci number. However, there are two factors that could affect this assignment strategy. On the one hand, the distributions of lengths at birth and at division are known to have coefficients of variation of 13.5 per cent [28], and consequently so do the cell sizes at any given cell age. And on the other, replication proteins can be assembled into a pre-replication structure that would lead to more foci than replicating forks [31]. Both effects could explain the number of $\mathrm{NrdB}$ foci found in the period between termination and initiation shown in Figure 3 where no replication forks are expected. Nevertheless, the good agreement found between the number of immunolabeled fluorescent NrdB foci per cell cycle period and the number predicted for replication protein foci provides clear support to sustain the association of the RNR with the replication fork. 


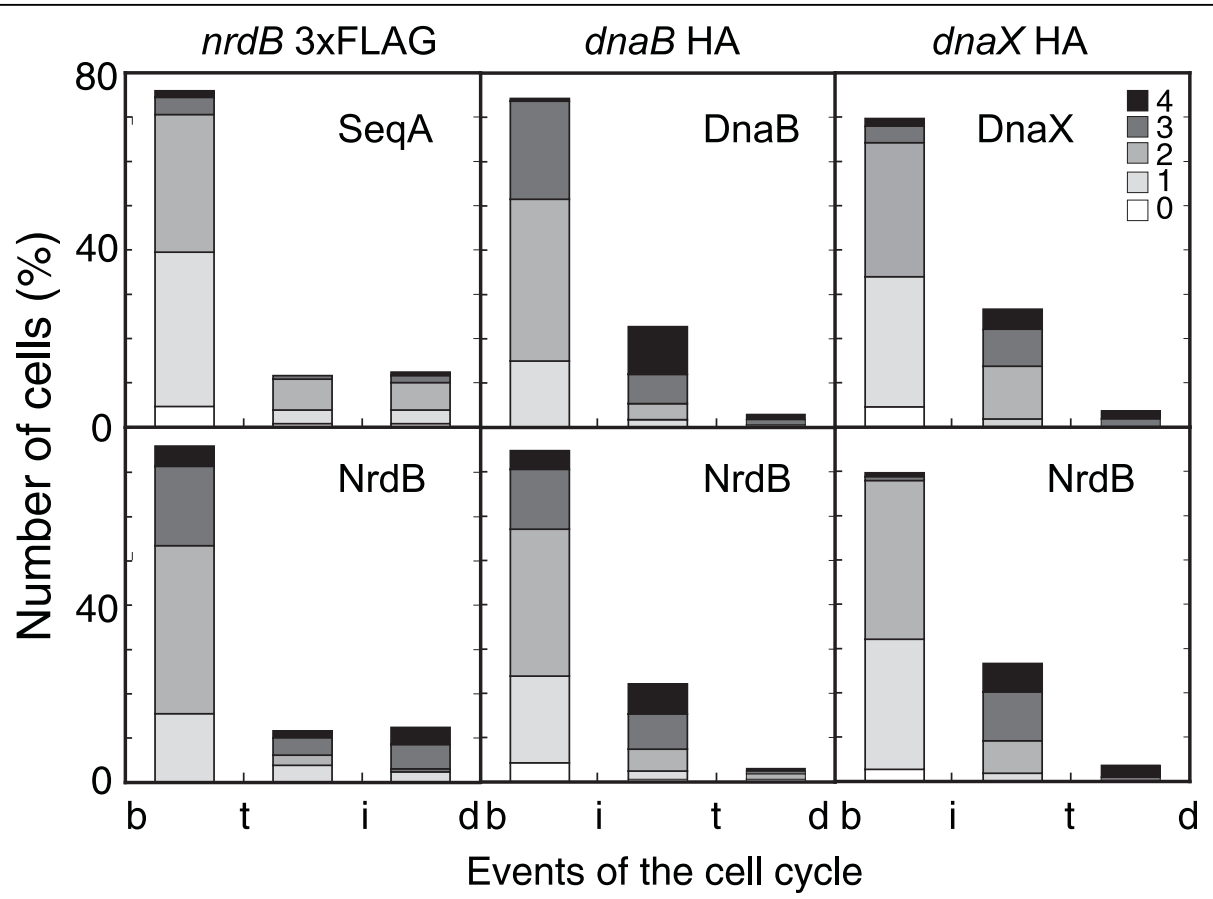

Figure $7 \mathrm{NrdB}$ foci and replication proteins foci along the cell cycle. Percentage of cells containing different numbers of foci during the three cell cycle periods described in Figure 1. Foci of fluorescent proteins were from SeqA and NrdB in strain CMT931 (nrdB::3 $\times$ FLAG); DnaB helicase and NrdB in strain CMT935 (nrdB::3XFLAG dnaB::HA); and DnaX $\tau$ subunit and NrdB in strain CMT936 (nrdB::3xFLAG dnaX::HA). Numbers of cells analyzed were 129,172, and 110, respectively. The inset shows the colour code for the number of foci per cell.

A prediction of this association would be that $\mathrm{NrdB}$ foci should decrease after replication ends. The presence of $\mathrm{NrdB}$ foci during the replication time and their decline after termination of replication was verified by fluorescence microscopy of a culture synchronized for initiation of chromosome replication. The treatment used provides reasonable synchronization of one chromosome replication round, but not all cells initiate replication. Moreover, after replication should have terminated, a number of cells were still replicating, most likely due to a low elongation rate of the $d n a C 2$ mutant strain at the high temperature [30]. This drawback prevents the disappearance of all replication forks, and could be the source of cells with NrdB foci after $90 \mathrm{~min}$ at the high temperature. A fraction of this number of non-replicating cells containing $\mathrm{NrdB}$ foci could also be

Table 1 Mean inter-foci distances $(\mu \mathrm{m})$ between tagged replication proteins

\begin{tabular}{llll}
\hline Proteins & Mean & St. Dev. & Number of foci-pairs measured \\
\hline NrdB SeqA & 0.13 & 0.10 & 82 \\
NrdB DnaB & 0.25 & 0.22 & 217 \\
NrdB DnaX & 0.15 & 0.13 & 141 \\
SeqA DnaB & 0.16 & 0.16 & 65 \\
SeqA DnaX & 0.15 & 0.14 & 68 \\
\hline
\end{tabular}

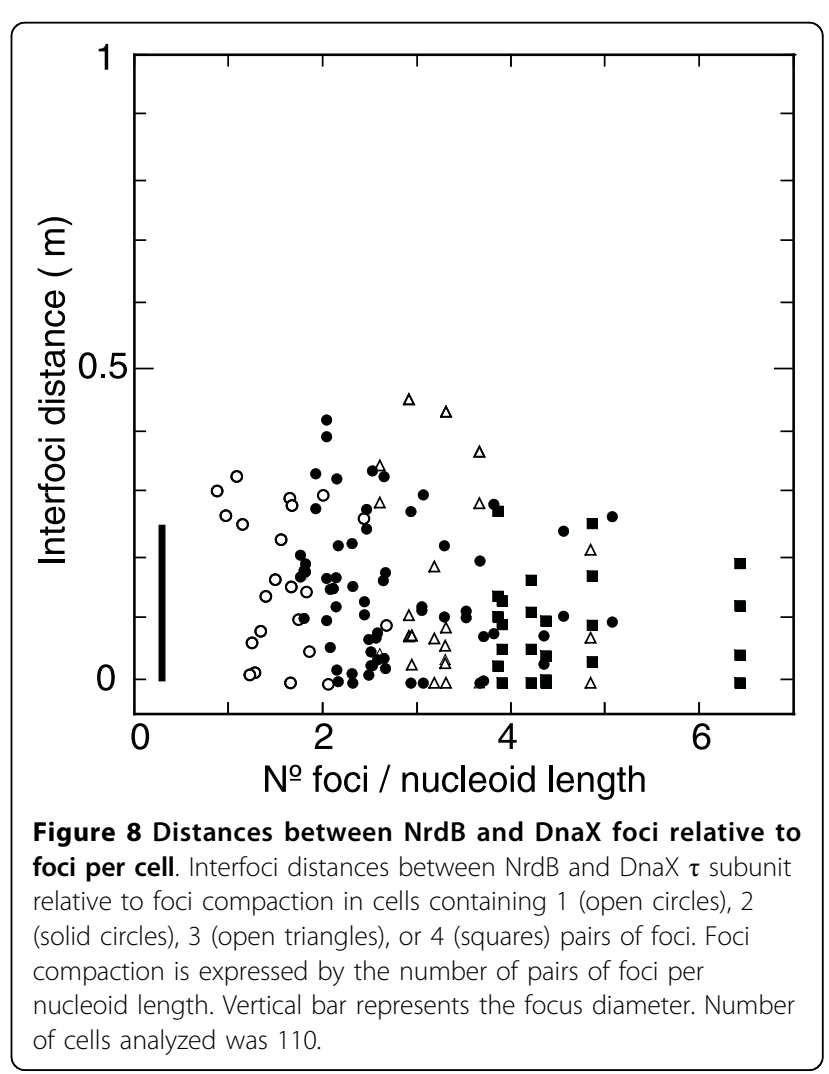


due to the aforementioned pre-assembly of the replication complexes.

In order to ascertain that RNR protein was indeed associated with the replication fork, we investigated the number of fluorescence immunolabeled NrdB foci together with foci from a C-terminal HA-tagged DnaB helicase, or from a C-terminal HA-tagged DnaX $\tau$ subunit from the DNA polymerase holoenzyme, or from immunolabeled fluorescent SeqA. Both measurements of foci per cell in the whole culture and per individual cell cycle period in the three strains gave very similar values for any of all three replication protein foci as well as for NrdB foci (Figures 6 and 7).

The greater number of DnaB helicase and DnaX $\tau$ subunit foci relative to those of SeqA could be explained by the pre-replication assembly of the replisome proteins [31]. Likewise, the greater number of $\mathrm{NrdB}$ foci relative to those of SeqA can be explained by assuming that this protein was also pre-assembled together with the replisome proteins before replication initiated. This might indicate that the whole replication hyperstructure could be organized some time before replication is initiated. This explanation could account for the number of CMT931 cells found without SeqA foci but with NrdB foci.

An implication of the association between RNR and the replisome would be the nearness of RNR to any replisome protein. We determined the distance between NrdB foci and those of SeqA, DnaB helicase, and DnaX $\tau$ subunit. The data showed that almost all distances found between $\mathrm{NrdB}$ foci and any other replication protein foci were the same as the distances between DnaB or $\tau$ foci and SeqA foci. Furthermore, our data show that these distances are not due to random localization corresponding to foci compaction. Therefore, we can conclude that $\mathrm{NrdB}$ protein is localized near the replication fork.

The above data lead to three important conclusions: (i) NrdB, and consequently RNR, is located in discrete foci. (ii) The number of NrdB foci per cell is the same as the predicted number of replication forks, and it is the same as the number of foci of the replisome proteins DnaB helicase and DNA polymerase III $\tau$ subunit. (iii) The localization of $\mathrm{NrdB}$ foci is not random; instead they are close to SeqA, DnaB, and DnaX $\tau$ subunit. With these results, the only convincing explanation is that RNR protein is associated with the replication complex. This association would predict that other proteins from the dNTP synthesizing complex could also be related to the replication fork, most likely in a higher order structure, the replication hyperstructure. High local concentrations of molecules can be attained by co-localization of proteins, and new functions can emerge [33]. Such organization in a replication hyperstructure might permit channeling and the compartmentation of precursors so as to provide a balanced supply of the four dNTPs at the concentration required for high speed processive chromosome replication.

\section{Conclusions}

NrdB protein, and most likely RNR protein, is assembled in clusters associated with nucleoids. The observations that $(i)$ the number of $\mathrm{NrdB}$ foci correlates with the number of replication forks in any cell cycle period, (ii) the end of replication causes the disappearance of most of the NrdB foci, (iii) the number of NrdB foci is very similar to that of SeqA, DnaB, and DnaX foci in any cell cycle period, and (iv) interfoci distances between $\mathrm{NrdB}$ and the three replication protein foci are very similar to those between replication proteins, allow us to conclude that $\mathrm{NrdB}$ clusters emerge and are related to the replisome during the bacterial chromosome replication period. These results provide clear support for the replication hyperstructure hypothesis.

\section{Methods}

\section{Bacterial strains, plasmids and growth conditions}

All strains used were derived from E. coli $\mathrm{K}-12$ (Table 2). CMT931, a CM735 containing $3 \times$ FLAG-tagged $n r d B$ gene, was used to immunolocalize the R2 subunit of RNR. Strains CMT935 and CMT936, containing HAtagged $d n a B$ gene and HA-tagged dnaX gene, respectively, and $3 \times$ FLAG-tagged $n r d B$ gene, were used for colocalization of DnaB helicase and subunit $\tau$ of DNA polymerase holoenzyme and RNR protein. For their construction, strain CMT927, a pKD46-harbouring CM735, was used. Strain EBO193 is CM735 $\Delta$ seqA and was used for nonspecific absorption of antibody antiSeqA. Bacteria were incubated at $37^{\circ} \mathrm{C}$ in $\mathrm{M} 9$ minimal glycerol medium supplemented with requirements. When required, kanamycin was used at $25 \mu \mathrm{g} \mathrm{ml}^{-1}$. Strain CMT934 (dnaC2 nrdB::3×FLAG) was used for immunolocalization of RNR in synchronized cells.

Plasmid pKD46 is a temperature sensitive replicon expressing $\lambda$ red genes under the arabinose-inducible ParaBAD promoter [34] and was used for linear DNA transformation. Plasmid pSUB11 and plasmid pSU314 were used as template for the amplification of DNA fragments containing the $3 \times$ FLAG and the influenza virus HA epitope sequences, respectively [35]. pSUB11 and pSU314 carry chloramphenicol- and kanamycinresistance genes, respectively, flanked by the recognition sites (FRT sites) of the yeast FLP recombinase in direct repeats [36].

\section{Construction of epitope-tagged proteins}

$3 \times$ FLAG epitope was attached to the C-terminal sequence of the R2 subunit of RNR, and HA epitope was tagged to the $\mathrm{C}$-terminus of DnaB helicase and of 
Table 2 Escherichia coli K-12 strains used in this work

\begin{tabular}{lll}
\hline Strain & \multicolumn{1}{c}{ Genotype } & Source \\
\hline CM735 & metE46 trp3 his4 thi1 galK2 lacY1 mtl1 ara9 tsx3 ton1 rps8 supE44 I- & Dr K. Skarstad \\
CMT927 & CM735/pKD46 & This work \\
CMT931 & CMT927 nrdB::3×FLAG & This work \\
CMT934 & CMT931 dnaC2 thr::Tn10 & This work \\
CMT935 & CMT931 dnaB::HA & This work \\
CMT936 & CMT931 dnaX::HA & This work \\
EBO193 & CM735 $\triangle$ seqA & Dr K. Skarstad \\
\hline
\end{tabular}

DNA polymerase III holoenzyme $\tau$ subunit, essentially as described $[34,35]$. Briefly, a DNA molecule containing the epitope-encoding sequence and the kanamycin- or chloramphenicol-resistance gene were amplified by PCR. For protein tagging, primers shown in Table 3 were used. Forward primers contained 36, 39, and 33 bases from downstream of the gene to be tagged and 20 bases (underlined) from the upstream sequence of the epitope-encoding sequence; reverse primers contained 39, 35 , and 35 bases from the downstream sequence following the end of the gene to be tagged, and 20 bases (underlined) from the 3 '-end of the FRT sequence downstream of the kanamycin or cloramphenicol gene. In this approach genes are not replaced, and $3 \times$ FLAG or HA were inserted in wild-type genes through homologous recombination after the last $\mathrm{C}$-terminal amino acid.

Strain CMT927 expressing $\lambda$ red functions was transformed with linear DNA obtained by PCR. A final 1 $\mathrm{mM} \mathrm{L}$-arabinose was used for the induction of the promoter. Kanamycin or chloramphenicol resistant transformants were selected in selective plates at $30^{\circ} \mathrm{C}$. Bacterial chromosomes containing 3'-terminal-tagged genes were verified by PCR and tagged proteins by western blot using the same antibodies as in the fluorescence microscopy (see below).

\section{Immunofluorescence microscopy}

Cell preparation and staining were performed as described [37]. Samples of $1.5 \mathrm{ml}$ from an exponentially growing culture were withdrawn at $0.15 \mathrm{OD}_{550}$, and cells were collected by centrifugation, washed, resuspended in $1 \mathrm{ml} \mathrm{TE}$ buffer, and fixed by adding the same volume of cold $74 \%$ ethanol.

Immunostaining of SeqA protein was performed using cell extracts from strain EBO193 for absorption of nonspecific SeqA antibody as described previously [7].

Ethanol-fixed cells $(100 \mu \mathrm{l})$ were stained with mouse monoclonal anti-FLAG M2-Cy3 antibody (SigmaAldrich) to immunolocalize RNR, or with mouse monoclonal anti-HA (clone 7) antibody (Sigma-Aldrich) and a secondary anti-mouse antibody conjugated to Alexa 488 (Molecular Probes) for DnaB helicase and $\tau$ subunit localization. When both antibodies were used in the same preparation, anti-FLAG antibody was used as the last treatment and after extensive PBS washing. SeqA was labeled with rabbit polyclonal anti-SeqA antibody and an anti-rabbit secondary antibody conjugated to FITC (Sigma-Aldrich), as previously described [24]. Immunostained cells were stained with Hoechst 33258, $1.5 \mathrm{mg} \mathrm{ml}^{-1}$, in $10 \mu \mathrm{l}$ mounting medium (40\% glycerol in $0.02 \mathrm{M}$ phosphate buffered saline, $\mathrm{pH}$ 7.5). $20 \mu \mathrm{l}$ of ethanol-fixed cells was spread onto a poly-L-lysinecoated slide, and dried at room temperatures. Slides of stained samples were stored at room temperature in the dark.

Images were captured with a Delta Vision optical microscope (Applied Precision) equipped with a $100 \times$ UPLS Apo objective and filter sets for DAPI, FI-GF-BF, and RD-TR-PE filters, and were deconvolved using 15 iterations of the Delta Vision constrained iterative deconvolution software (Applied Precision). From every field, different micrographs using each of the three filters were taken. The deconvolved images were saved in TIFF format and imported into ImageJ software for cell and nucleoid size measurements and localization of foci centres (Wayne Rasband, Research Services Branch, National Institute of Mental Health, USA).

\section{Cell cycle parameters}

Growth rates of exponentially growing bacteria were determined by the absorbance of the cultures at 550 $\mathrm{nm}$. For the cell cycle parameter measurements, cells were treated with rifampicin, $150 \mu \mathrm{g} \mathrm{ml}^{-1}$, and cephalexin, $50 \mu \mathrm{g} \mathrm{ml}^{-1}$, to inhibit initiation of replication and cell division, respectively. After three hours of treatment, cells were fixed, and the number of chromosomes per cell was measured by flow cytometry using a FAC Star DIVA (Becton Dickinson) instrument, essentially as previously described [37]. The time required for chromosome replication, $C$, and the time from the termination of replication to cell division, $D$, were determined from the number of cells before and after the initiation of replication in the initiation time equations $[38,39]$.

Briefly, $C$ can be obtained from the average amount of DNA per cell obtained by cytometry after the rifampicin and cephalexin treatment relative to that amount before the treatment $(d G)$. This relative increment in the 
Table 3 Primers used for protein tagging

\begin{tabular}{lll}
\hline Tagged protein & Primer direction & Primer sequence \\
\hline NrdB & forward & GAAGTGGACACCGACGATTTGAGTAACAACCAGCTCGACTACAAAGACCATGACGG \\
& reverse & TCAAATTITCCAATCGCCACTAATTGTTCCATGCACATCATATGAATATCCTCCTTAG \\
\hline DnaB & forward & CGCTTCGACAACTATGCGGGGCCGCAGTACGACGACGAATTCTATCCGTATGATGTTCC \\
& reverse & GTGTTCCTTGATAAGTGTTGCTTTAATTACCTAATATATGAATATCCTCCTTAG \\
\hline DnaX & forward & GCGGAGCTGGATGAAGAAAGTATCCGCCCCATTTCTATCCGTATGATGTTCC \\
& reverse & GGTTCTCTCTCAATCACGTTAAGGATGACGAACGTATATGAATATCCTCCTTAG \\
\hline
\end{tabular}

Underlined bases in forward primers correspond to the upstream of the epitope-encoding sequence.

Underlined bases in reverse primers correspond to the $3^{\prime}$-end of the FRT sequence downstream of the kanamycin or chloramphenicol gene.

amount of DNA per cell is: $d G=\left(2^{n} n \ln 2\right) /\left(2^{n}-1\right)$, and $n=C / \tau$ [40]. If we designate $R=(C+D) / \tau$ and $r$ being the integer value of $R$, analysis of bacterial cell cycles with any possible overlapping results in a number of origins per cell before and after initiation of replication equal to $2^{r}$ and $2^{(r+1)}$ respectively. These number of origins correspond to the number of full replicated chromosomes per cell at the end of the rifampicin and cephalexin treatment. The time at wich replication initiates, $t_{i}$, can be deduced to be $t_{i}=1+r-R$. The frequency of cells before and after the initiation of replication can be deduced from Powell's age distribution [38] to be $2-2^{(R-r)}$ and $2^{(R-r)}-1$ respectively, and are obtained experimentally by flow cytrometry. The length of $D$ period can be obtained by using flow cytometry data in these equations.

\section{Bacterial synchronization for initiation of chromosome replication}

Synchronization of bacteria for one initiation of chromosome replication was performed as described [30]. Strain CMT934 was grown in glycerol minimal medium at $30^{\circ} \mathrm{C}$. At mid log phase, the culture was transferred to $42^{\circ} \mathrm{C}$ for $1 \mathrm{~h}$ to inhibit initiation and complete ongoing rounds of replication. Cells were subsequently shifted to $30^{\circ} \mathrm{C}$ to allow one initiation event, and after 6 min they were transferred back to $42^{\circ} \mathrm{C}$ to avoid further initiations. Incorporation of ${ }^{3} \mathrm{H}$-thymidine into TCA-precipitable material showed that this treatment gave rise to one replication round and that it terminated after about 50 min (data not shown).

\footnotetext{
Acknowledgements

We are very grateful to Drs J. Jiménez and R. Daga of the Centro Andaluz de Biología del Desarrollo (Seville, Spain) for the use of the Delta Vision microscope, and to Dr K. Skarstad for anti-SeqA antibody and the use of the flow cytometer. We thank Dr J. Casadesus for plasmids pKD46, pSUB11, and pSU314, and Encarna Ferrera for her excellent technical help. This work was supported by grants from the J. Extremadura and MEC (Spain). MAS-R acknowledges the studentship from J. Extremadura.
}

\section{Authors' contributions}

MASR performed experiments, and helped to design experiments and to draft the manuscript. FM conceived and designed experiments, conducted and analyzed cytometry experiments, and helped to draft the manuscript.
AJS conceived the study, its design and coordination, and drafted the manuscript. All authors read and approved the final manuscript.

Received: 23 June 2009

Accepted: 26 January 2010 Published: 26 January 2010

\section{References}

1. Marians KJ: Understanding how the replisome works. Nat Struct Mol Biol 2008, 15:125-127.

2. Eklund $H$, Uhlin U, Färnegardh M, Logan DT, Nordlund P: Structure and function of the radical enzyme ribonucleotide reductase. Prog Biophys Mol Biol 2001, 77:177-268.

3. Nordlund P, Reichard P: Ribonucleotide reductases. Annu Rev Biochem 2006, 75:681-706.

4. Mathews CK, Sinha NK: Are DNA precursors concentrated at replication sites?. Proc Natl Acad Sci USA 1982, 79:302-306.

5. Warner HR: Properties of ribonucleoside disphosphate reductase in nucleotide-permeable cells. J Bacterio/ 1973, 115:18-22.

6. Buckstein $\mathrm{MH}, \mathrm{He} \mathrm{J}$, Rubin $\mathrm{H}$ : Characterization of nucleotide pools as a function of physiological state in Escherichia coli. J Bacteriol 2008, 190:718-726.

7. Reichard P: Control of deoxyribonucleotide synthesis in vitro and in vivo. Adv Enzyme Reg 1972, 10:3-16.

8. Reddy GP, Mathews CK: Functional compartmentation of DNA precursors in T4 phage-infected bacteria. J Biol Chem 1978, 253:3461-3467.

9. Butland G, Peregrín-Alvarez JM, Li J, Yang W, Yang X, Canadien V, Starostine A, Richards D, Beattie B, Krogan N, Davey M, Parkinson J, Greenblatt J, Emili A: Interaction network containing conserved and essential protein complexes in Escherichia coli. Nature 2005, 433:531-537.

10. Chao J, Leach M, Karam J: In vivo functional interaction between DNA polymerase and dCMP-hydroxymethylase of bacteriophage T4. J Virology 1977, 24:557-563.

11. Kim J, Wheeler $L$, Shen $R$, Mathews CK: Protein-DNA interactions in the T4 dNTP synthetase complex dependent on gene 32 single-stranded DNA-binding protein. Mol Microbiol 2005, 55:1502-1514.

12. Reichard P: Interactions between deoxyribonucleotide and DNA synthesis. Ann Rev Biochem 1988, 57:349-374.

13. Mathews CK, Slabaugh MB: Eukaryotic DNA metabolism: Are deoxyribonucleotides channeled to replication sites?. Exp Cell Res 1986, 162:285-295

14. Reddy GPV, Pardee AP: Multienzyme complex for metabolic channeling in mammalian DNA replication. Proc Natl Acad Sci USA 1980, 77:3312-3316.

15. Guzmán EC, Caballero JL, Jiménez-Sánchez A: Ribonucleoside diphosphate reductase is a component of the replication hyperstructure in Escherichia coli. Mol Microbiol 2002, 43:487-495.

16. Fuchs JA, Karlstrom HO, Warner HR, Reichard P: Defective gene product in dnaF mutant of E. coli. Nature 1972, 238:69-71.

17. Guarino E, Jiménez-Sánchez A, Guzmán EC: Defective ribonucleoside diphosphate reductase impairs replication fork progression in Escherichia coli. J Bacteriol 2007, 189:3496-3501.

18. Riola J, Guarino E, Guzmán EC, Jiménez-Sánchez A: Differential inhibition of NDP reductase by chemical inactivation and by the thermosensitive mutation nrdA101 in Escherichia coli. Cell Mol Biol Letters 2007, 12:70-81.

19. Norris V, Blaauwen T, Cabin-Flaman A, Doi RH, Errington J, Harshey R, Janniere L, Jiménez-Sánchez A, Jin DJ, Levin PA, Mileykovskaya E, Minsky A, 
Saier M, Skarstad K: A Taxonomy of Bacterial Hyperstructures. Annu Rev Microbiol 2007, 61:309-329.

20. Norris V, Blaauwen T, Cabin-Flaman A, Doi RH, Errington J, Harshey R, Janniere L, Jiménez-Sánchez A, Jin DJ, Levin PA, Mileykovskaya E, Minsky A, Saier M, Skarstad K: Functional Taxonomy of Bacterial Hyperstructures. Microbiol Mol Biol Rev 2007, 71:230-253.

21. Molina F, Skarstad K: Replication fork and SeqA focus distributions in Escherichia coli suggest a replication hyperstructure dependent on nucleotide metabolism. Mol Microbiol 2004, 52:1597-1612.

22. Herrick J, Sclavi B: Ribonucleotide reductase and the regulation of DNA replication: an old story and an ancient heritage. Mol Microbiol 2007, 63:22-34.

23. Watt RM, Wang J, Leong M, Kung H, Cheah KSE, Liu D, Danchin A, Huang JD: Visualizing the proteome of Escherichia coli an efficient and versatile method for labeling chromosomal coding DNA sequences (CDSs) with fluorescent protein genes. Nucleic Acids Res 2007, 35(e37):1-11.

24. Hiraga S, Ichinose C, Niki H, Yamazoe M: Cell cycle-dependent duplication and bidirectional migration of SeqA-associated DNA-protein complexes in E. coli. Mol Cell 1998, 1:381-387.

25. Hiraga S: Dynamic localization of bacterial and plasmid chromosomes. Ann Rev Genet 2000, 34:21-59.

26. Molina F, Sánchez-Romero MA, Jiménez-Sánchez A: Dynamic organization of replication forks into factories in Escherichia coli. Process Biochem 2008, 43:1171-1177.

27. Morigen, Odsbu I, Skarstad K: Growth rate dependent numbers of SeqA structures organize the multiple replication forks in rapidly growing Escherichia coli. Genes Cells 2009, 14:643-657.

28. Kubitschek HE, Woldringh $\mathrm{CL}$ : Cell elongation and division probability during the Escherichia coli growth cycle. J Bacteriol 1983, 153:1379-1387.

29. Trueba FJ, Koppes LJ: Exponential growth of Escherichia coli B/r during its division cycle is demonstrated by the size distribution in liquid culture. Arch Microbiol 1998, 169:491-496.

30. Yamazoe M, Adachi S, Kanaya S, Ohsumi K, Hiraga S: Sequential binding of SeqA protein to nascent DNA segments at replication forks in synchronized cultures of Escherichia coli. Mol Microbiol 2005, 55:289-298,

31. Den Blaauwen T, Aarsman MEG, Wheeler L, Nanninga N: Pre-replication assembly of E. coli replisome components. Mol Microbiol 2006, 62:695-708.

32. Wang $X$, Reyes-Lamothe R, Sherratt DJ: Visualizing genetic loci and molecular machines in living bacteria. Biochem Soc Trans 2008, 36:749-753.

33. Müller-Hill B: What is life? The paradigm of DNA and protein cooperation at high local concentrations. Mol Microbiol 2006, 60:253-255.

34. Datsenko KA, Wanner BL: One-step inactivation of chromosomal genes in Escherichia coli K-12 using PCR products. Proc Natl Acad Sci USA 2000, 97:6640-6645.

35. Uzzau S, Figueroa-Bossi N, Rubino S, Bossi L: Epitope tagging of chromosomal genes in Salmonella. Proc Natl Acad Sci USA 2001, 98:15264-15269.

36. Cherepanov PP, Wackernagel W: Gene disruption in Escherichia coli: TcR and $\mathrm{KmR}$ cassettes with the option of Flp-catalyzed excision of the antibiotic-resistance determinant. Gene 1995, 158:9-14.

37. Fossum S, Soreide S, Skarstad K: Lack of SeqA focus formation specific DNA binding and proper protein multimerization in the Escherichia coli sequestration mutant seqA2. Mol Microbiol 2003, 47:619-632.

38. Powell EO: Growth rate and generation time of bacteria, with special reference to continuous culture. J Gen Microbiol 1956, 15:492-511.

39. Wold S, Skarstad S, Steen HB, Stokke T, Boye E: The initiation mass for DNA replication in Escherichia coli $\mathrm{K}-12$ is dependent on growth rate. EMBO J 1994, 13:2097-2102.

40. Sueoka N, Yoshikawa H: The chromosome of Bacillus subtilis. Theory of marker frequency analysis. Genetics 1965, 52:747-757.

doi:10.1186/1471-2199-11-11

Cite this article as: Sánchez-Romero et al:: Correlation between ribonucleoside-diphosphate reductase and three replication proteins in Escherichia coli. BMC Molecular Biology 2010 11:11.

\section{Submit your next manuscript to BioMed Central and take full advantage of:}

- Convenient online submission

- Thorough peer review

- No space constraints or color figure charges

- Immediate publication on acceptance

- Inclusion in PubMed, CAS, Scopus and Google Scholar

- Research which is freely available for redistribution

Submit your manuscript at www.biomedcentral.com/submit
Biomed Central 